\title{
Micro- and nanoscale electrical characterization of large-area graphene transferred to functional substrates
}

\author{
Gabriele Fisichella ${ }^{1,2}$, Salvatore Di Franco ${ }^{1}$, Patrick Fiorenza ${ }^{1}$, \\ Raffaella Lo Nigro ${ }^{1}$, Fabrizio Roccaforte ${ }^{1}$, Cristina Tudisco ${ }^{3}$, \\ Guido G. Condorelli ${ }^{3}$, Nicolò Piluso ${ }^{1}$, Noemi Spartà ${ }^{4}$, Stella Lo Verso ${ }^{4}$, \\ Corrado Accardi ${ }^{4}$, Cristina Tringali ${ }^{4}$, Sebastiano Ravesi ${ }^{4}$ \\ and Filippo Giannazzo*1
}

\section{Full Research Paper}

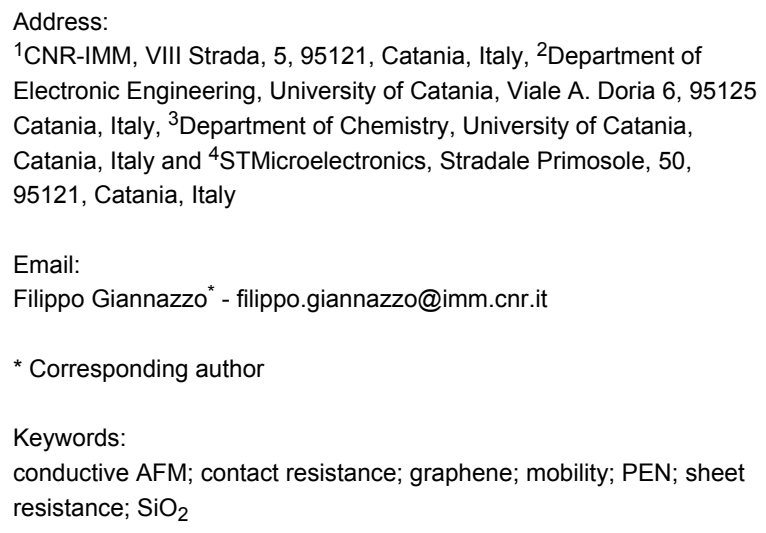

${ }^{1}$ CNR-IMM, VIII Strada, 5, 95121, Catania, Italy, ${ }^{2}$ Department of Electronic Engineering, University of Catania, Viale A. Doria 6, 95125 Catania, Italy, ${ }^{3}$ Department of Chemistry, University of Catania, Catania, Italy and ${ }^{4}$ STMicroelectronics, Stradale Primosole, 50, 95121, Catania, Italy

Email:

Filippo Giannazzo* - filippo.giannazzo@imm.cnr.it

* Corresponding author

Keywords:

conductive AFM; contact resistance; graphene; mobility; PEN; sheet resistance; $\mathrm{SiO}_{2}$

\author{
Beilstein J. Nanotechnol. 2013, 4, 234-242. \\ doi:10.3762/bjnano.4.24 \\ Received: 17 November 2012 \\ Accepted: 07 March 2013 \\ Published: 02 April 2013 \\ This article is part of the Thematic Series "High-resolution electrical and \\ chemical characterization of nm-scale organic and inorganic devices". \\ Guest Editors: E. Meyer and P. Eyben
}

(c) 2013 Fisichella et al; licensee Beilstein-Institut.

License and terms: see end of document.

\begin{abstract}
Chemical vapour deposition (CVD) on catalytic metals is one of main approaches for high-quality graphene growth over large areas. However, a subsequent transfer step to an insulating substrate is required in order to use the graphene for electronic applications. This step can severely affect both the structural integrity and the electronic properties of the graphene membrane. In this paper, we investigated the morphological and electrical properties of CVD graphene transferred onto $\mathrm{SiO}_{2}$ and on a polymeric substrate (poly(ethylene-2,6-naphthalene dicarboxylate), briefly PEN), suitable for microelectronics and flexible electronics applications, respectively. The electrical properties (sheet resistance, mobility, carrier density) of the transferred graphene as well as the specific contact resistance of metal contacts onto graphene were investigated by using properly designed test patterns. While a sheet resistance $R_{\mathrm{sh}} \approx 1.7 \mathrm{k} \Omega / \mathrm{sq}$ and a specific contact resistance $\rho_{\mathrm{c}} \approx 15 \mathrm{k} \Omega \cdot \mu \mathrm{m}$ have been measured for graphene transferred onto $\mathrm{SiO}_{2}$, about $2.3 \times$ higher $R_{\text {sh }}$ and about $8 \times$ higher $\rho_{\mathrm{c}}$ values were obtained for graphene on PEN. High-resolution current mapping by torsion resonant conductive atomic force microscopy (TRCAFM) provided an insight into the nanoscale mechanisms responsible for the very high $\rho_{\mathrm{c}}$ in the case of graphene on PEN, showing a ca. 10× smaller "effective" area for current injection than in the case of graphene on $\mathrm{SiO}_{2}$.
\end{abstract}




\section{Introduction}

Graphene is the single layer of graphite and can be described as a $2 \mathrm{D}$ crystal of $\mathrm{sp}^{2}$ hybridised carbon atoms in a honeycomb lattice [1]. Its electrical and optical characteristics are mainly related to the peculiar energy band structure, i.e., to the linear dispersion relation and to the zero band gap. For neutral (undoped) graphene the Fermi level is coincident with the Dirac point, that is, the intersection point between the valence and the conduction band. From these properties originate the high intrinsic field-effect mobility [2-4] of graphene, its high thermal conductivity [5], and its optical transparency [6].

Due to its excellent mobility, graphene has been proposed as a channel material in high-frequency devices operating in the $100 \mathrm{GHz}$ to terahertz range [7]. Thanks to the very high specific capacitance, it is an excellent candidate for fabricating highly efficient supercapacitors [8]. Furthermore, the unique combination between high optical transparency $(\approx 97 \%)$ in a wide range of wavelengths (from near IR to near UV), high conductivity, and excellent flexibility, make it the ideal candidate as a transparent electrode for flat-panel displays, for OLEDs, and for the next generation of flexible organic solar cells $[9,10]$.

Currently, the most used method of graphene production for basic studies is the mechanical exfoliation of graphite [1], which was the first method to obtain graphene under ambient laboratory conditions. This method yields graphene fragments of excellent crystalline quality, but of small size $(1-100 \mu \mathrm{m})$ and which are randomly distributed on the substrate. Carrier mobilities $>10^{5} \mathrm{~cm}^{2} \cdot \mathrm{V}^{-1} \cdot \mathrm{s}^{-1}$ have been measured on exfoliated graphene flakes suspended between electrodes [4], whereas values from 10,000 to $30,000 \mathrm{~cm}^{2} \cdot \mathrm{V}^{-1} \cdot \mathrm{s}^{-1}$ are obtained for flakes on common dielectric substrates [11]. As a matter of fact, future applications in large-scale electronics will require waferscale sheets of graphene that can be deterministically placed on a substrate.

Other methods, such as epitaxial graphene growth by controlled graphitization of silicon carbide [12-15] and by chemical vapour deposition (CVD) on catalytic metals [9], are more suitable for large-area applications, as has been demonstrated in the past few years.

Considering the case of CVD, the two main catalytic metals used for graphene growth are nickel and copper [16]. In the case of CVD growth on copper foils, due to the extremely low solubility of carbon in the solid metal, the graphene formation is purely a surface process and this allows one to obtain single-layer graphene on a very large fraction (above $90 \%$ ) of the metal surface [17]. In order to use CVD- grown graphene for electronic applications, the graphene membrane must be transferred to a properly chosen insulating substrate [18].

A commonly used method to transfer graphene grown on copper foil onto the target substrate is the use of a resist film deposited on the graphene surface, which is used as a support during the etching of the underlying $\mathrm{Cu}$ foil. After the etching process, the graphene membrane attached to the resist scaffold is mechanically attached to the target substrate and the resist is eliminated. There are two crucial points in this transfer technique: (i) promoting the adhesion of graphene onto the target substrate; and (ii) cleaning the transferred graphene from resist residues.

The first issue is especially critical, because a bad compatibility between graphene and the substrate typically causes the formation of macroscopic defects (cracks) or folding of the graphene membrane when placed onto the substrate. Several aspects can influence the surface adhesion between graphene and the substrate, including the substrate roughness and the surface energy. Though a complete understanding of this issue has not yet been achieved, it can be argued that, due to the inherent hydrophobic character of graphene, the adhesion of large-area membranes can be favoured on substrates with a similar hydrophobic character.

Resist and, more generally, polymeric residues adsorbed onto graphene are known to severely degrade graphene transport properties [19]. However, a complete cleaning of the transferred graphene from those residues is particularly difficult, because it requires thermal treatments at temperatures of up to $400{ }^{\circ} \mathrm{C}$ in vacuum [19] or under reducing $\left(\mathrm{N}_{2} / \mathrm{H}_{2}\right.$ or $\left.\mathrm{Ar} / \mathrm{H}_{2}\right)$ ambient conditions [20]. Such high thermal budgets are not compatible with some substrates of interest for future graphene electronics, such as the flexible polymeric ones.

In this paper, the transfer and electrical properties of CVDgrown graphene on different substrates have been addressed. In particular, two substrates of interest for electronic applications were taken into consideration:

1. $\mathrm{SiO}_{2}$ (300 nm thick) thermally grown on $\mathrm{Si}$, for its largescale use in microelectronics;

2. poly(ethylene-2,6-naphthalene dicarboxylate) or PEN, a transparent polymer analogue to the more common PET, but with stronger mechanical resistance, higher degradation temperature and higher chemical inertness in acid and alkaline conditions, which can be useful for transparent and flexible electronic applications. 
The electronic properties of the transferred graphene have been characterized both at the macro- and nanoscale, by using properly fabricated test patterns and conductive atomic force microscopy, respectively. This characterization provided an insight into the different electronic properties of graphene transferred to the two kinds of substrates.

\section{Graphene growth and transfer}

Graphene was grown by CVD on ca. $25 \mu \mathrm{m}$ thick polycrystalline copper foils at a temperature of $1000{ }^{\circ} \mathrm{C}$ by using $\mathrm{CH}_{4} / \mathrm{H}_{2}$ as precursors. In Figure 1a an optical microscopy image of the $\mathrm{Cu}$ surface after graphene growth is reported, showing the typical size of $\mathrm{Cu}$ grains, ranging from about 20 to $200 \mu \mathrm{m}$. The graphene membrane, uniformly covering the $\mathrm{Cu}$ foil, is mostly composed of a single layer of graphene (over $90 \%$ of the surface area), while bilayers or multilayers can be typically found at $\mathrm{Cu}$ grain boundaries. A representative Raman spectrum on the $\mathrm{Cu}$ surface is reported in Figure 1b, showing the characteristic $G$ peak (at $\approx 1580 \mathrm{~cm}^{-1}$ ) and $2 \mathrm{D}$ peak (at $\approx 2640 \mathrm{~cm}^{-1}$ ) of the graphitic material. In particular, the high ratio of the $2 \mathrm{D}$ versus $\mathrm{G}$ peak and the symmetric character of the 2D peak (fitted by a single Lorentzian component with FWHM $\approx 38 \mathrm{~cm}^{-1}$, as shown in the insert of Figure $1 \mathrm{~b}$ ) are consistent with the presence of a single layer of graphene. The D peak (at $\approx 1320 \mathrm{~cm}^{-1}$ ) indicates the presence of a certain density of defects in the as-grown material. Morphology and phase images of as-grown graphene on $\mathrm{Cu}$, obtained by tapping mode atomic force microscopy (AFM), are also reported in Figure 1c and Figure 1d. In particular, from the phase image it is possible to see the presence of peculiar corrugations (wrinkles) in the graphene membrane over the copper foil. The origin of these corrugations will be discussed in the following.

\section{Graphene transfer onto silicon dioxide}

The graphene membrane was transferred from the $\mathrm{Cu}$ foil onto a $\mathrm{Si}$ wafer coated by $300 \mathrm{~nm}$ thick thermally grown $\mathrm{SiO}_{2}$. This oxide thickness was properly selected because it ensures the best optical contrast between bare $\mathrm{SiO}_{2}$ regions and regions coated by the monoatomic thick membrane, due to an effect of constructive optical interference [21].

Since as-grown $\mathrm{SiO}_{2}$ typically exhibits a hydrophilic behaviour (as shown by contact-angle measurements, yielding values of $10 \pm 2^{\circ}$ ), before graphene transfer proper surface treatments were performed to achieve a partially hydrophobic character (with contact-angle values of $52 \pm 2^{\circ}$ ). The chemical status of the $\mathrm{SiO}_{2}$ surface before graphene transfer was also characterized by X-ray photoelectron spectroscopy (XPS) measurements.

In Figure $2 \mathrm{a}$ the optical image of a large-area $\left(\mathrm{cm}^{2}\right)$ graphene membrane transferred onto $\mathrm{SiO}_{2}$ is shown. Due to the good optical contrast between the graphene-coated and bare $\mathrm{SiO}_{2}$ areas, a homogenous graphene membrane, free from macroscopic cracks, can be observed. Higher resolution morphological analyses of the graphene layer onto $\mathrm{SiO}_{2}$ were carried out by tapping mode AFM. Two representative AFM images at different magnifications are reported in Figure $2 b$ and Figure 2c. As evident from Figure 2b, a high density of submi-

(a)

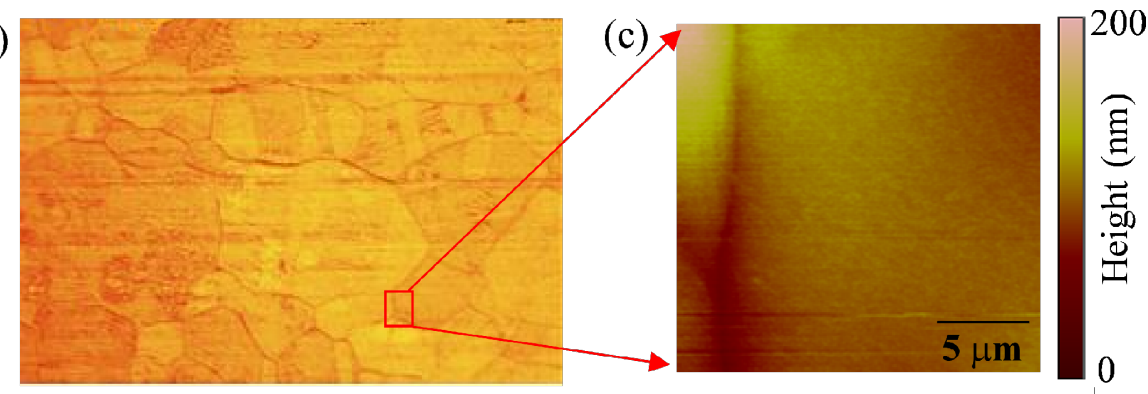

(b) 110

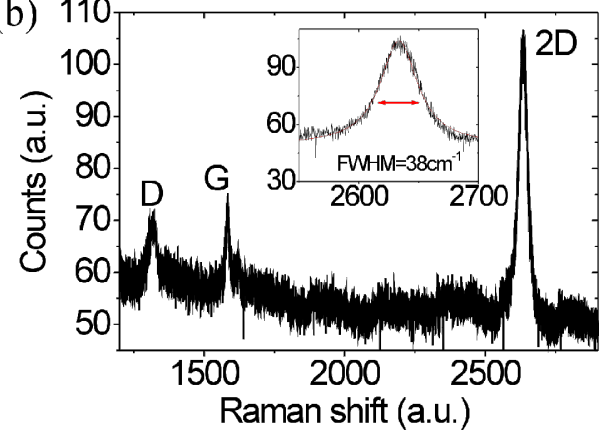

(d)

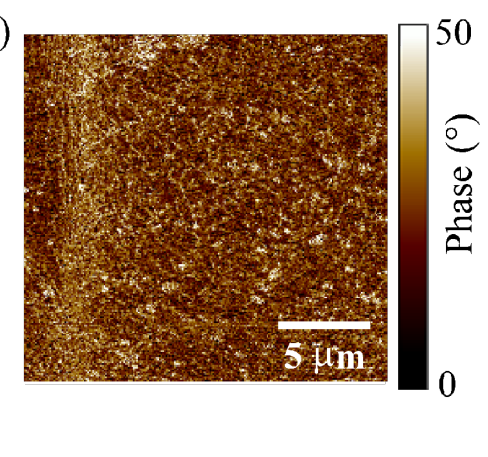

Figure 1: As-grown graphene on a copper foil: (a) Optical image, (b) Raman Spectroscopy, (c) AFM morphology and (d) phase. 
crometric features can be observed on the graphene, mainly represented by contaminations (polymer residues) left after removal of the thick resist film employed for the transfer process. Furthermore, some typical defects of the graphene membrane, such as small cracks or peculiar corrugations (wrinkles), are indicated in Figure 2b. While the first kind of defect is mostly related to the mechanical handling of graphene during transfer, wrinkles can be present also in the as-grown graphene on $\mathrm{Cu}$ (as already shown in Figure 1d). Corrugations in as-grown graphene originate from the cooling-down step of the CVD process, due to the different thermal expansion coefficients between graphene and $\mathrm{Cu}$. However, some of the wrinkles can also be produced during the transfer process to the substrate. In Figure $2 \mathrm{~d}$ the height profile of a wrinkle is displayed, whereas in Figure 2e the step height between graphene and bare $\mathrm{SiO}_{2}$ on a crack region extracted from Figure $2 \mathrm{c}$ is shown. The measured step height $(\approx 0.8 \mathrm{~nm})$ is consistent with the typical values reported by AFM for a single layer of graphene on $\mathrm{SiO}_{2}$ [22]. Both cracks and corrugations contribute to the degradation of the electronic transport properties in graphene [23].

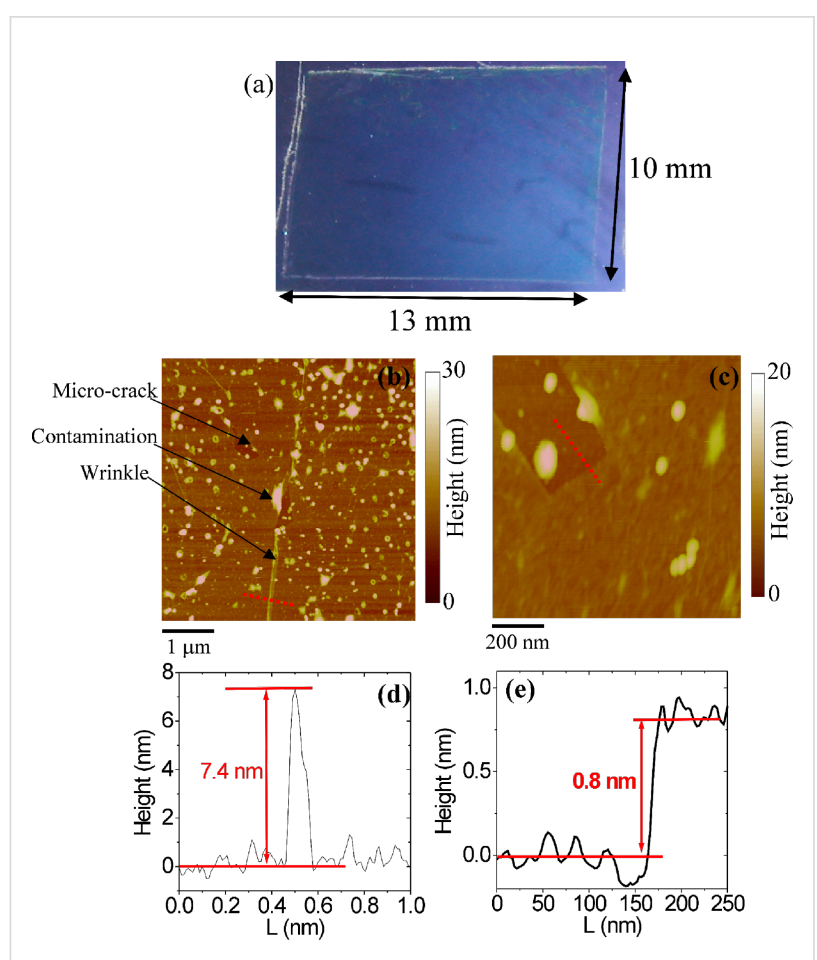

Figure 2: Optical image of a $(13 \times 10) \mathrm{mm}^{2}$ graphene membrane transferred onto $\mathrm{SiO}_{2}(\mathrm{a})$, and AFM morphologies at $(5 \times 5) \mu \mathrm{m}^{2}(\mathrm{~b})$ and $(1 \times 1) \mu \mathrm{m}^{2}(\mathrm{c})$ magnifications. Line scans on a peculiar corrugation of the graphene membrane $(d)$ and across a microscopic $\operatorname{crack}(\mathrm{e})$.

\section{Graphene transfer onto PEN}

In contrast to the case of virgin $\mathrm{SiO}_{2}$, which is naturally hydrophilic and requires proper treatments to be converted into a hydrophobic surface, contact-angle measurements on the as-received PEN substrate typically yield high values of the contact angle $\left(\approx 80^{\circ}\right)$, indicating the highly hydrophobic character of this surface. A representative morphological image of the PEN substrate is reported in Figure 3a, showing a high surface roughness (RMS $\approx 6.3 \mathrm{~nm}$ ). When transferred onto PEN, graphene conformally covers the substrate morphology. By carefully comparing Figure $3 b$ on graphene-coated PEN with Figure 3a on bare PEN, the presence of a pleated and wrinkled membrane superimposed on the rough substrate can be deduced. The higher roughness value in graphene-coated PEN can also be partially ascribed to the presence of resist residues from the transfer process.

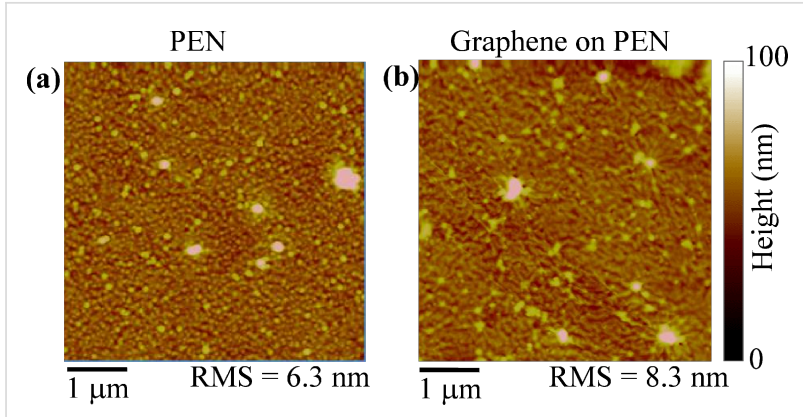

Figure 3: Tapping-mode AFM images of the bare PEN surface (a) and of graphene transferred onto PEN (b).

The homogeneity of graphene membranes on the transparent PEN substrate can be characterized in a straightforward way by optical transmittance microscopy, since a contrast between graphene-coated and uncoated regions arises from the finite absorbance $(\approx 2.7 \%$ ) of the graphene monolayer. Optical images (not reported) demonstrate the absence of macroscopic cracks and fractures in the graphene transferred onto PEN, which can be explained as a consequence of a very good compatibility between the two materials.

\section{Results and Discussion Microscale electrical characterization}

The electronic transport properties of the large-area graphene transferred onto the two different substrates have been characterized on the macroscopic scale by electrical measurements on transmission line model (TLM) test structures. An optical microscopy image of a TLM test pattern fabricated in graphene on $\mathrm{SiO}_{2}$ is reported in Figure 4a. It consists of a set of metal contacts $(\mathrm{Ni} / \mathrm{Au})$ with identical geometry (width $W=200 \mu \mathrm{m}$ and length $L=100 \mu \mathrm{m}$ ) and different spacing, $d$, deposited onto a laterally insulated rectangular graphene area. The current-voltage $(I-V)$ characteristics for different distances between adjacent contacts are reported in Figure 4b, showing an Ohmic behaviour for all the contact distances. In Figure $4 \mathrm{c}$ the 

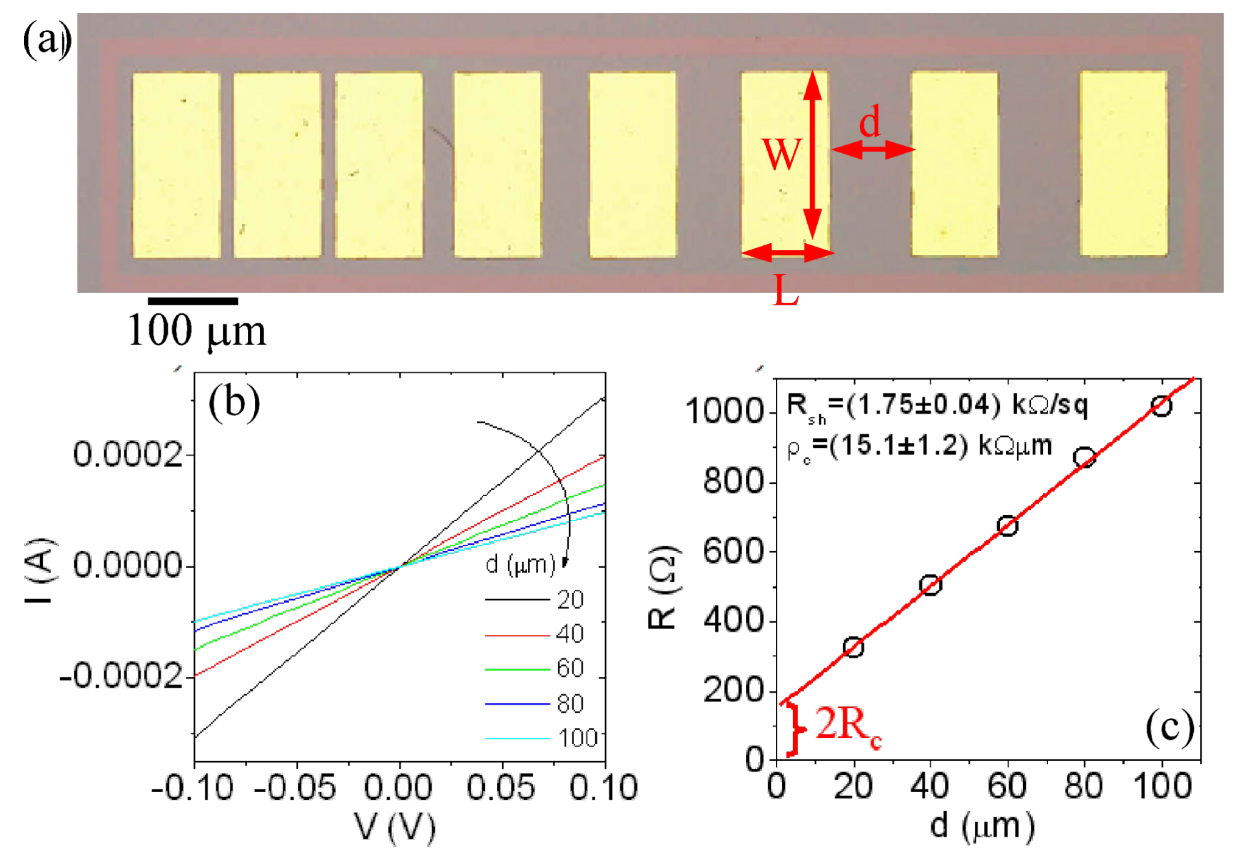

Figure 4: (a) Optical Image of a TLM structure, (b) $I-V$ characteristics measured between pairs of contacts at different distances and (c) extracted resistance plotted versus distance. From the linear fit of $R$ versus $d$, the sheet resistance and the specific contact resistance were evaluated.

resistance $R$, obtained from the slope of each curve, is plotted versus the contact distance. According to the TLM theory [24], $R$ is related to the metal/graphene contact resistance $R_{\mathrm{c}}$ and to the graphene sheet resistance $R_{\mathrm{sh}}$ according to the relation

$$
R=2 R_{\mathrm{c}}+\frac{R_{\mathrm{sh}}}{W} d
$$

By linear fitting of the data in Figure 4c by Equation 1, the sheet resistance $\left(R_{\mathrm{sh}}=1.75 \pm 0.04 \mathrm{k} \Omega / \mathrm{sq}\right)$ and the contact resistance $\left(R_{\mathrm{c}}=75 \pm 6 \Omega\right)$ contributions have been determined. Since $R_{\mathrm{c}}$ clearly depends on the pad size, the specific contact resistance $\rho_{\mathrm{c}}=R_{\mathrm{c}} \cdot \mathrm{W}$ normalized to the contact width was also evaluated, obtaining a value $\rho_{\mathrm{c}}=15.1 \pm 1.2 \mathrm{k} \Omega \cdot \mu \mathrm{m}$.

For graphene transferred onto the $\mathrm{SiO}_{2}(300 \mathrm{~nm}) / \mathrm{Si}$ substrate, the $\mathrm{n}^{+}$-doped Si substrate can be employed as global back-gate (see schematic in Figure 5a) to induce an electrostatic shift of the Fermi level of graphene and, hence, to tune the carrier density of the material. In Figure $5 b$ the resistance versus the distance between adjacent contacts is reported for different values of the back-gate bias $V_{\mathrm{g}}$ from -40 to $40 \mathrm{~V}$. By linear fitting of each curve, the dependence of the specific contact resistance $\left(\rho_{\mathrm{c}}\right)$ and of the sheet resistance $\left(R_{\mathrm{sh}}\right)$ on the gate bias was extracted (see Figure $5 \mathrm{c}$ and Figure $5 \mathrm{~d}$, respectively). It is worth noting that both $R_{\mathrm{sh}}$ and $\rho_{\mathrm{c}}$ exhibit a monotonically increasing behaviour with the back-gate bias values in the considered bias range.
Compared to the typically ambipolar behaviour observed in back-gated FET devices fabricated in graphene exfoliated from HOPG onto $\mathrm{SiO}_{2}$ (which exhibit hole conduction for negative gate bias and electron conduction for positive bias) [10,11], a p-type doping can be deduced from the electrical characterization of CVD-grown graphene membranes transferred onto $\mathrm{SiO}_{2}$. This doping can probably be ascribed to the adsorbed resist impurities left after transfer. Furthermore, the measured $\rho_{c}$ is almost one order of magnitude higher than in the case of the same nickel-gold contacts on graphene exfoliated onto $\mathrm{SiO}_{2}$ [25].

The hole conductance $\sigma=1 / R_{\mathrm{sh}}$ in graphene is related to the hole mobility $\mu_{\mathrm{p}}$ and density $p$ by the following relation

$$
\sigma=q \mu_{\mathrm{p}} p=q \mu_{\mathrm{p}}\left(\frac{C_{\mathrm{ox}} V_{\mathrm{g}}}{q}+p_{0}\right)
$$

where $p$ has been expressed as the sum of $p_{0}$, i.e., the doping at $V_{\mathrm{g}}=0$, and of the doping induced by the back-gate bias $\left(C_{\mathrm{ox}} \cdot V_{\mathrm{g}} / q\right)$, with $q$ being the electron charge and $C_{\mathrm{ox}}=\varepsilon_{0} \varepsilon_{\mathrm{ox}} / t_{\mathrm{ox}}$ the oxide capacitance per unit area.

By linear fitting of the experimental $\sigma$ versus $V_{\mathrm{g}}$ data with Equation 2 (see insert of Figure $5 \mathrm{~d}$ ), the values of $\mu_{\mathrm{p}}=$ $793 \pm 18 \mathrm{~cm}^{2} \cdot \mathrm{V}^{-1} \cdot \mathrm{s}^{-1}$ and $p_{0}=(4.4 \pm 0.1) \times 10^{12} \mathrm{~cm}^{-2}$ have 

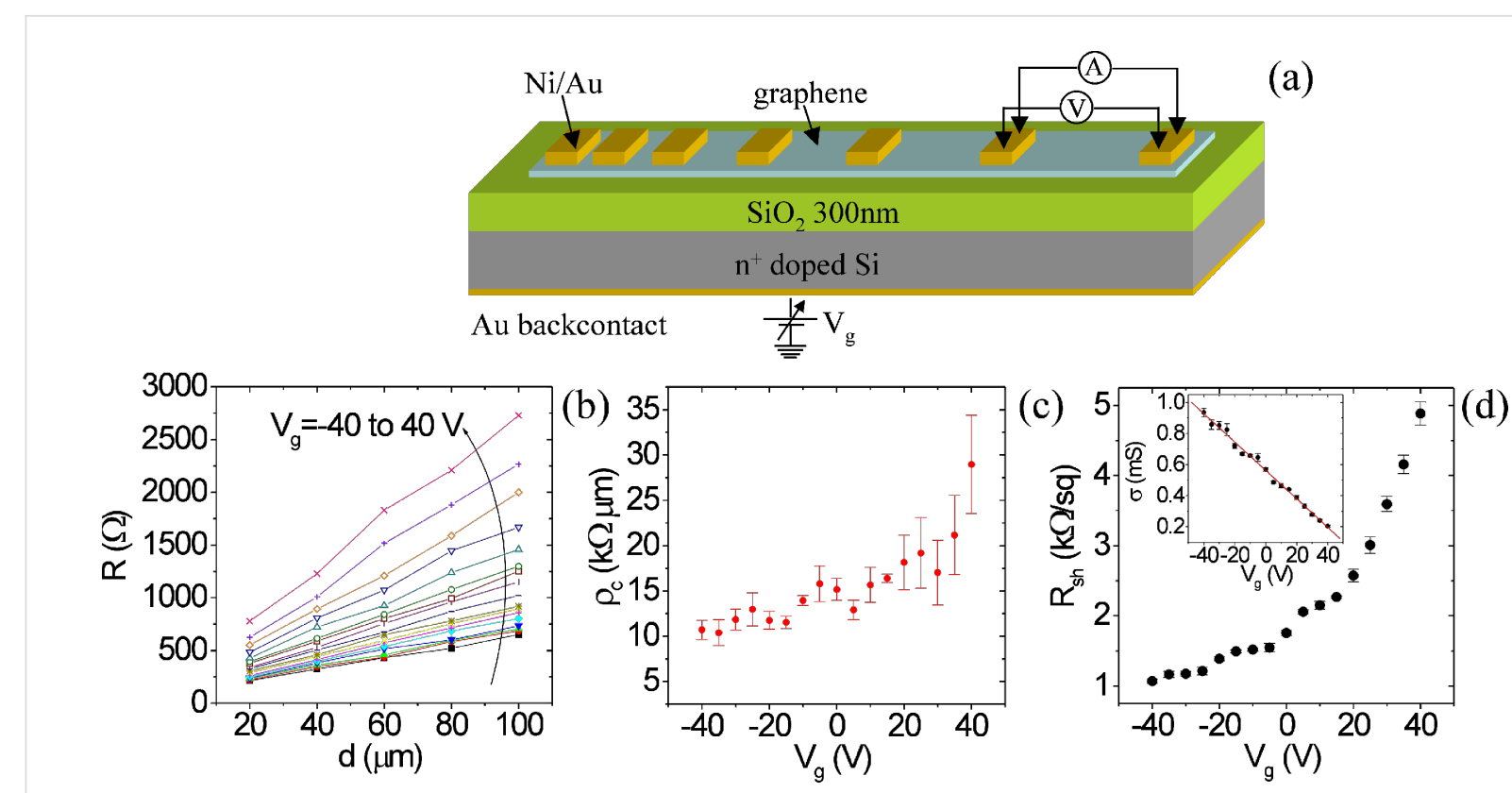

Figure 5: (a) Schematic representation of the back-gated TLM device. (b) Resistance versus distance between adjacent contacts for different $V_{\mathrm{g}}$ values from -40 to $40 \mathrm{~V}$. Extracted specific contact resistance $\rho_{\mathrm{c}}(\mathrm{c})$ and sheet resistance $R_{\mathrm{sh}}(\mathrm{d})$ versus $V_{\mathrm{g}}$. The insert in (d) displays the linear fit of the conductance data to extract the hole density and mobility in graphene.

been obtained. It is worth noting that the effect of traps at the graphene $/ \mathrm{SiO}_{2}$ interface is not accounted for in the determination of $\mu_{\mathrm{p}}$ from Equation 2. This approximation implies an overestimation of the hole density $p$, which is actually reduced with respect to the value induced by the field effect due to carrier trapping, and, consequently, an underestimation of the carrier mobility. Furthermore, the presence of charged traps at the graphene/substrate interface strongly affects the mobility in graphene due to Coulomb scattering $[11,26]$, leading to a degradation with respect to the ideal value in the absence of interface traps.

A similar macroscopic electrical characterization using TLM structures was performed also in CVD graphene transferred onto PEN. In this case, the sheet resistance and specific contact resistance only were measured, whereas an estimate of mobility and carrier density was not feasible due to the absence of a back gate. A comparison between the $R_{\mathrm{sh}}$ and $\rho_{\mathrm{c}}$ values for graphene on the two substrates (obtained with a back-gate bias $V_{\mathrm{g}}=0$ for graphene on $\mathrm{SiO}_{2}$ and without a back-gate bias for graphene on $\mathrm{PEN}$ ) is reported in Table 1.

It is worth noting that the $R_{\mathrm{sh}}$ of graphene on PEN is about 2.3× higher than on $\mathrm{SiO}_{2}$, whereas the $\rho_{\mathrm{c}}$ is about $8 \times$ higher. Since the same CVD graphene was used for both samples and similar transfer quality has been achieved on both substrates, these electrical differences can be ascribed to the different kind of interaction between graphene and $\mathrm{SiO}_{2}$ and graphene and PEN.

Table 1: Comparison between the sheet resistance and the metal/
graphene specific contact resistance of graphene deposited on $\mathrm{SiO}_{2}$
and on PEN.
\[ \mathrm{SiO}_{2} \]

In fact, a van der Waals interaction occurs between graphene and $\mathrm{SiO}_{2}$, whereas it cannot be excluded that other kind of bonds occur locally between graphene and the polymeric substrate, leading to a partial $\mathrm{sp}^{3}$ hybridization of graphene $\mathrm{C}$ atoms and, hence, to a local disruption of graphene electronic properties. This idea is supported by the presence of a certain density of defects in the initial graphene (as shown by Raman measurements), that can represent preferential sites for bonding with the polymeric chains.

\section{Nanoscale electrical characterization}

In order to get a deeper insight into the mechanisms leading to the different electronic properties of transferred graphene on the two substrates, and, in particular, to the very different specific contact resistance values, the local electrical properties of graphene on $\mathrm{SiO}_{2}$ and on PEN were characterized by torsion resonance conductive AFM (TRCAFM).

TRCAFM is an evolution of the more widely used contact mode conductive atomic force microscopy (CAFM). It is a dynamic 
scanning probe method based on a conductive tip scanned at close proximity $(0.3-3.0 \mathrm{~nm})$ to the sample surface, while oscillating in the torsional mode. The torsion amplitude is used as the feedback signal to measure surface morphology.

A dc bias was applied to a macroscopic metal contact deposited onto graphene, and the current locally injected from the nanometric conductive tip into graphene was probed by a high sensitivity (fA) current sensor connected to the tip. In this way, TRCAFM combines the high resolution of dynamic scanning probe microscopy for morphological mapping with the ability for nanoscale-resolution current mapping of CAFM. This operation mode has been demonstrated to be particularly useful to perform high-resolution morphology and current maps in graphene [27,28].

Figure $6 \mathrm{a}$ and Figure $6 \mathrm{~b}$ show the surface morphology and current map for graphene on $\mathrm{SiO}_{2}$. In Figure $6 \mathrm{c}$ and Figure $6 \mathrm{~d}$ the histograms of the height and of the current values extracted from the two maps are reported, respectively. Similarly, Figure 6e and Figure 6f show the morphology and current maps in graphene on PEN, while Figure $6 \mathrm{~g}$ and Figure $6 \mathrm{~h}$ show the derived histograms of height and current values. As evident from this comparison, the morphology of graphene on $\mathrm{SiO}_{2}$ is much flatter than the morphology of graphene on PEN, due to the very different roughness of the substrates. The most interesting aspect is represented by the comparison of the current maps and of the current histograms. Clearly, both histograms exhibit two peaks, but it is worth noting that the integrated percentage of counts under the higher conductivity peak is much higher for graphene on $\mathrm{SiO}_{2}(85 \%)$ than for graphene on PEN (9\%). This striking difference indicates that in the case of graphene on
$\mathrm{SiO}_{2}$ most of the area contributes to current injection from the tip to graphene, whereas in the case of graphene on PEN only a small fraction of the area contributes to the current injection. This observation is in close agreement with the difference in the specific contact resistance values obtained from macroscopic TLM measurements. The physical origin of this difference is still the subject of investigation.

\section{Conclusion}

In conclusion, the transfer of CVD-grown graphene onto different substrates $\left(\mathrm{SiO}_{2}\right.$ and $\left.\mathrm{PEN}\right)$ and its morphological and electrical properties have been investigated in detail. Using TLM test patterns the electrical properties (sheet resistance, mobility, carrier density) of the transferred graphene and the specific contact resistance of metal contacts on graphene were determined. While a sheet resistance $R_{\text {sh }} \approx 1.7 \mathrm{k} \Omega / \mathrm{sq}$ and a specific contact resistance $\rho_{\mathrm{c}} \approx 15 \mathrm{k} \Omega \cdot \mu \mathrm{m}$ were measured (at $V_{\mathrm{g}}=0 \mathrm{~V}$ ) for graphene transferred onto $\mathrm{SiO}_{2}$, about $2.3 \times$ higher $R_{\mathrm{sh}}$ and about $8 \times$ higher $\rho_{\mathrm{c}}$ values were obtained for graphene on PEN. High-resolution current mapping by torsion resonant conductive atomic force microscopy (TRCAFM) revealed a ca. $10 \times$ smaller "effective" area for current injection in the case of graphene on PEN than in the case of CVD graphene transferred on $\mathrm{SiO}_{2}$, which is consistent with higher $\rho_{\mathrm{c}}$ values. These electrical differences could be ascribed also to the different kind of interaction of graphene with $\mathrm{SiO}_{2}$ and PEN. While a van der Waals bond occurs between graphene and $\mathrm{SiO}_{2}$, other kind of bonds can be locally formed between graphene and the polymeric chains of PEN, leading to a partial $\mathrm{sp}^{3}$ hybridization of graphene and, hence, to a local modification of its electronic properties.
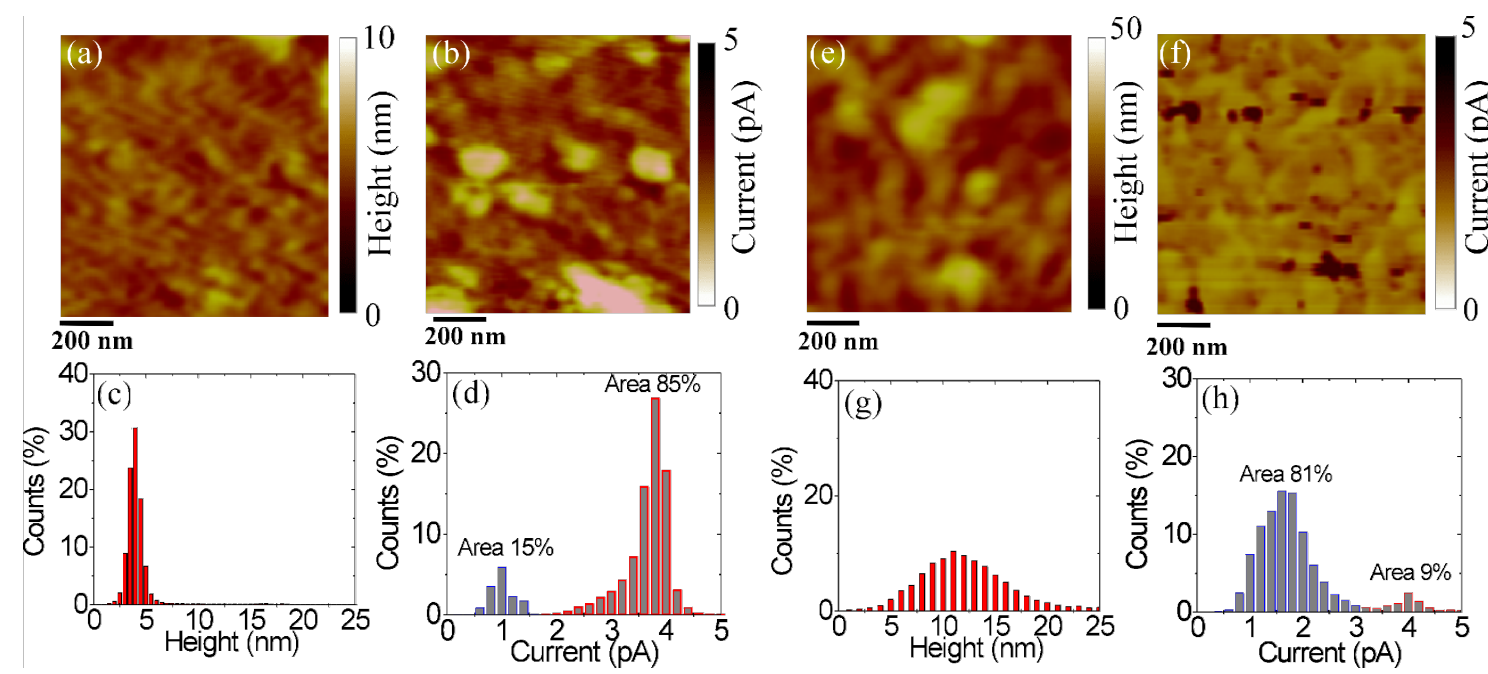

Figure 6: TRCAFM of Graphene on $\mathrm{SiO}_{2}$ : (a) morphology and (c) the related histogram, (b) current map and (d) the related histogram. TRCAFM of Graphene on PEN: (a) morphology and (c) the related histogram, (b) current map and (d) the related histogram. 


\section{Experimental}

Tapping mode AFM and TRCAFM: Both tapping-mode AFM and torsion resonant conductive atomic force microscopy (TRCAFM) measurements were performed by using a DI3100 microscope with Nanoscope V electronics. For TRCAFM, we used Pt/Ir-coated Si tips with an apex radius of curvature of $10 \mathrm{~nm}$, which were driven in torsional motion at a frequency of $940 \mathrm{kHz}$.

TLM fabrication and electrical characterization: The transmission line model (TLM) test patterns were fabricated on the transferred graphene membranes by using the following procedure. First, rectangular graphene areas were isolated from the external membrane by lithographically defining and opening a rectangular frame in a hard mask resist and by performing graphene etching of the frame by $\mathrm{O}_{2}$ plasma treatments. Subsequently, a set of nickel-gold rectangular contacts were deposited by sputtering and defined by the lift-off method. The contacts had identical geometry $(200 \mu \mathrm{m}$ width and $100 \mu \mathrm{m}$ length $)$ and the distance between the pairs of adjacent contacts were 20,20, $40,60,80,100$ and $100 \mu \mathrm{m}$, respectively. The current-voltage $(I-V)$ characteristics were measured in a Karl-Süss probe station by using a HP 4156B parameter analyzer.

Raman spectroscopy: Raman measurements were performed by using a Horiba-Jobin Yvon spectrometer. Spectra were collected in the backscattering configuration with $a \approx 633 \mathrm{~nm}$ laser source and a $100 \times$ objective, focusing the excitation light to $\mathrm{a} \approx 1 \mu \mathrm{m}$ spot.

XPS: $\mathrm{SiO}_{2}$ atomic surface composition was determined by $\mathrm{X}$-ray photoelectron analysis (XPS) by using a PHI ESCA/SAM 5600 Multitechnique spectrometer. XPS experiments were carried out with a base pressure of $2 \times 10^{-10}$ torr. A monochromated $\mathrm{Al} \mathrm{K} \alpha$ radiation source $(\mathrm{h} v=1486.6 \mathrm{eV})$ was used, and XPS spectra were collected at various photoelectron angles (relative to the sample surface) in the $20-45^{\circ}$ range.

\section{Acknowledgements}

The authors want to thank S. Rizzolo and M. Cannas from the Department of Physics of University of Palermo for participation in the sample characterization and for useful discussions, respectively. This work was supported, in part, by the GraphicRF project funded by ESF.

\section{References}

1. Novoselov, K. S.; Geim, A. K.; Morozov, S. V.; Jiang, D.; Zhang, Y.; Dubonos, S. V.; Grigorieva, I. V.; Firsov, A. A. Science 2004, 306, 666-669. doi:10.1126/science.1102896
2. Morozov, S. V.; Novoselov, K. S.; Katsnelson, M. I.; Schedin, F.; Elias, D. C.; Jaszczak, J. A.; Geim, A. K. Phys. Rev. Lett. 2008, 100, 016602. doi:10.1103/PhysRevLett.100.016602

3. Chen, J.-H.; Jang, C.; Xiao, S.; Ishigami, M.; Fuhrer, M. S. Nat. Nanotechnol. 2008, 3, 206-209. doi:10.1038/nnano.2008.58

4. Bolotin, K. I.; Sikes, K. J.; Jiang, Z.; Klima, M.; Fudenberg, G.; Hone, J.; Kim, P.; Stormer, H. L. Solid State Commun. 2008, 146, 351-355. doi:10.1016/j.ssc.2008.02.024

5. Balandin, A. A.; Ghosh, S.; Bao, W.; Calizo, I.; Teweldebrhan, D.; Miao, F.; Lau, C. N. Nano Lett. 2008, 8, 902-907. doi:10.1021/nl0731872

6. Nair, R. R.; Blake, P.; Grigorenko, A. N.; Novoselov, K. S.; Booth, T. J.; Stauber, T.; Peres, N. M. R.; Geim, A. K. Science 2008, 320, 1308. doi:10.1126/science.1156965

7. Lin, Y.-M.; Dimitrakopoulos, C.; Jenkins, K. A.; Farmer, D. B.; Chiu, H.-Y.; Grill, A.; Avouris, P. Science 2010, 327, 662. doi:10.1126/science.1184289

8. Stoller, M. D.; Park, S.; Zhu, Y.; An, J.; Ruoff, R. S. Nano Lett. 2008, 8, 3498-3502. doi:10.1021/nl802558y

9. Kim, K. S.; Zhao, Y.; Jang, H.; Lee, S. Y.; Kim, J. M.; Kim, K. S.; Ahn, J.-H.; Kim, P.; Choi, J.-Y.; Hong, B. H. Nature 2009, 457, 706-710. doi:10.1038/nature07719

10. Bonaccorso, F.; Sun, Z.; Hasan, T.; Ferrari, A. C. Nat. Photonics 2010 , 4, 611-622. doi:10.1038/nphoton.2010.186

11. Giannazzo, F.; Sonde, S.; Lo Nigro, R.; Rimini, E.; Raineri, V. Nano Lett. 2011, 11, 4612-4618. doi:10.1021/nl2020922

12. Emtsev, K. V.; Bostwick, A.; Horn, K.; Jobst, J.; Kellogg, G. L.; Ley, L.; McChesney, J. L.; Ohta, T.; Reshanov, S. A.; Röhrl, J.; Rotenberg, E.; Schmid, A. K.; Waldmann, D.; Weber, H. B.; Seyller, T. Nat. Mater. 2009, 8, 203. doi:10.1038/nmat2382

13. Virojanadara, C.; Syväjarvi, M.; Yakimova, R.; Johansson, L. I.; Zakharov, A. A.; Balasubramanian, T. Phys. Rev. B 2008, 78, 245403. doi:10.1103/PhysRevB.78.245403

14. Vecchio, C.; Sonde, S.; Bongiorno, C.; Rambach, M.; Yakimova, R.; Raineri, V.; Giannazzo, F. Nanoscale Res. Lett. 2011, 6, 269. doi:10.1186/1556-276X-6-269

15. Giannazzo, F.; Raineri, V. Riv. Nuovo Cimento Soc. Ital. Fis. 2012, 35, 267-304. doi:10.1393/ncr/i2012-10076-7

16. Li, X.; Cai, W.; Colombo, L.; Ruoff, R. S. Nano Lett. 2009, 9 , 4268-4272. doi:10.1021/n1902515k

17. Li, X.; Cai, W.; An, J.; Kim, S.; Nah, J.; Yang, D.; Piner, R.; Velamakanni, A.; Jung, I.; Tutuc, E.; Banerjee, S. K.; Colombo, L.; Ruoff, R. S. Science 2009, 324, 1312. doi:10.1126/science.1171245

18. Kang, J.; Shin, D.; Bae, S.; Hong, B. H. Nanoscale 2012, 4, 5527-5537. doi:10.1039/c2nr31317k

19. Pirkle, A.; Chan, J.; Venugopal, A.; Hinojos, D.; Magnuson, C. W.; McDonnell, S.; Colombo, L.; Vogel, E. M.; Ruoff, R. S.; Wallace, R. M. Appl. Phys. Lett. 2011, 99, 122108. doi:10.1063/1.3643444

20. Kedzierski, J. T.; Hsu, P.-L.; Reina, A.; Kong, J.; Healey, P. D.; Wyatt, P. W.; Keast, C. L. IEEE Electron Device Lett. 2009, 30, 745-747. doi:10.1109/LED.2009.2020615

21. Giannazzo, F.; Sonde, S.; Raineri, V.; Patanè, G.; Compagnini, G.; Aliotta, F.; Ponterio, R.; Rimini, E. Phys. Status Solidi C 2010, 7, 1251-1255. doi:10.1002/pssc.200982967

22. Giannazzo, F.; Sonde, S.; Raineri, V.; Rimini, E. Nano Lett. 2009, 9 , 23. doi:10.1021/nl801823n

23. Zhu, W.; Low, T.; Perebeinos, V.; Bol, A. A.; Zhu, Y.; Yan, H.; Tersoff, J.; Avouris, P. Nano Lett. 2012, 12, 3431-3436. doi:10.1021/nl300563h 
24. Schroder, D. K. Semiconductor Material and Device Characterization, 3rd ed.; John Wiley \& Sons: Hoboken, NJ, 2006.

25. Venugopal, A.; Colombo, L.; Vogel, E. M. Appl. Phys. Lett. 2010, 96, 013512. doi: $10.1063 / 1.3290248$

26. Sonde, S.; Giannazzo, F.; Vecchio, C.; Yakimova, R.; Rimini, E.;

Raineri, V. Appl. Phys. Lett. 2010, 97, 132101. doi:10.1063/1.3489942

27. Sonde, S.; Giannazzo, F.; Raineri, V.; Yakimova, R.; Huntzinger, J.-R.; Tiberj, A.; Camassel, J. Phys. Rev. B 2009, 80, 241406.

doi:10.1103/PhysRevB.80.241406

28. Giannazzo, F.; Deretzis, I.; La Magna, A.; Roccaforte, F.; Yakimova, R. Phys. Rev. B 2012, 86, 235422. doi:10.1103/PhysRevB.86.235422

\section{License and Terms}

This is an Open Access article under the terms of the Creative Commons Attribution License

(http://creativecommons.org/licenses/by/2.0), which permits unrestricted use, distribution, and reproduction in any medium, provided the original work is properly cited.

The license is subject to the Beilstein Journal of

Nanotechnology terms and conditions:

(http://www.beilstein-journals.org/bjnano)

The definitive version of this article is the electronic one which can be found at: doi:10.3762/bjnano.4.24 